\title{
SELETIVIDADE DE INSETICIDAS A TRÊS VESPIDAE PREDADORES DE DIONE JUNO JUNO (LEPIDOPTERA: HELICONIDAE) ${ }^{1}$
}

\author{
MARCELO FIALHO DE MOURA ${ }^{2}$, MARCELO PICANÇO ${ }^{3}$, ALFREDO HENRIQUE ROCHA GONRING ${ }^{2}$ \\ e CLÁUDIOHORSTBRUCKNER ${ }^{4}$
}

\begin{abstract}
RESUMO - Dentre os insetos que atacam o maracujazeiro, Dione juno juno (Lepidoptera: Heliconidae) é considerada a praga-chave. Estudou-se a seletividade dos inseticidas fentiom, cartape, malatiom e deltametrina a Dione juno juno, em relação às vespas predadoras Polybia fastidiosuscula, Polybia scutellaris e Protonectarina sylveirae (Hymenoptera: Vespidae). Estimaram-se as curvas concentração-mortalidade e mediante o uso da concentração letal do inseticida em $90 \%$ dos indivíduos $\left(\mathrm{CL}_{90}\right)$ calcularam-se os índices de seletividade diferencial e índices de tolerância. A deltametrina foi seletiva à $P$. scutellaris e $P$. fastidiosuscula e medianamente seletiva à $P$. sylveirae e o cartape foi medianamente seletivo às três espécies de vespas predadoras. O malatiom foi seletivo a $P$. sylveirae e medianamente seletivo a $P$. fastidiosuscula. As vespas predadoras $P$. fastidiosuscula e $P$. scutellaris foram mais tolerantes a deltametrina e ao fentiom do que $P$. sylveirae, enquanto o $P$. fastidiosuscula e $P$. sylveirae toleraram mais o cartape do que $P$. scutellaris. O malatiom foi mais tolerado pela espécie $P$. sylveirae do que por P. fastidiosuscula e P. scutellaris.
\end{abstract}

Termos para indexação: maracujá, Passiflora edulis f. flavicarpa, lagarta-do-maracujá, Polybia fastidiosuscula, Polybia scutellaris, Protonectarina sylveirae.

\section{SELECTIVITY OF INSECTICIDES TO THREE VESPIDAE PREDATORS OF DIONE JUNO JUNO (LEPIDOPTERA: HELICONIDAE)}

\begin{abstract}
Among insects that attack passion fruit, Dione juno juno (Lepidoptera: Heliconidae) is considered the most dangerous plague. The selectivity of the insecticides fenthion, cartap, malathion and deltamethrin to the predatory wasps Polybia fastidiosuscula, Polybia scutellaris and Protonectarina sylveirae (Hymenoptera: Vespidae) was studied based on these insecticide toxicities to their prey Dione juno juno. Concentration-mortality regression lines were obtained and the estimated lethal concentration of insecticide to $90 \%\left(\mathrm{LC}_{90}\right)$ of the individuals were used for the calculation of the differential selectivity index and tolerance index. Deltamethrin was selective in favor of $P$. scutellaris and $P$. fastidiosuscula and showed intermediate selectivity to $P$. sylveirae, while cartap showed intermediate selectivity to all three species of predatory wasps. Malathion was selectivite to $P$. sylveirae and showed intermediate selectivity to $P$. fastidiosuscula. The predatory wasps $P$. sylveirae and P. fastidiosuscula were more tolerant to cartap than P. scutellaris and P. sylveirae was more tolerant to malathion than P. fastidiosuscula and P. scutellaris.
\end{abstract}

Index terms: Passion fruit, Passiflora edulis f. flavicarpa, passion fruit caterpillar, Polybia fastidiosuscula, Polybia scutellaris, Protonectarina sylveirae.

\section{INTRODUÇÃO}

O Brasil é o principal produtor mundial de maracujá-amarelo (Passiflora edulis f.flavicarpa Deg.),

\footnotetext{
${ }^{1}$ Aceito para publicação em 9 de março de 1999.

${ }^{2}$ Eng. Agrôn., Dep. de Biologia Animal, Universidade Federal de Viçosa (UFV), CEP 36571-000 Viçosa, MG.

${ }^{3}$ Eng. Agrôn., D.Sc., Dep. de Biologia Animal, UFV. E-mail: picanco@mail.ufv.br

${ }^{4}$ Eng. Agrôn., D.Sc., Dep. de Fitotecnia, UFV.
}

e supre $30 \%$ do consumo mundial de suco concentrado extraído de seus frutos. Dentre os insetos que atacam o maracujazeiro, Dione juno juno $\mathrm{Cr}$. (Lepidoptera: Heliconidae) é considerada a praga-chave. Suas lagartas causam desfolha, reduzindo o crescimento e produção do maracujazeiro, e ataques sucessivos desta praga podem causar a morte das plantas (Lima et al., 1994; Teixeira et al., 1994).

O controle de D. juno juno é realizado principalmente pela aplicação de inseticidas como cartape, 
deltametrina, fentiom e malatiom (Andrei, 1996; Picanço et al., 1996). O uso intensivo de inseticidas pode acarretar uma série de problemas, como a redução de populações de inimigos naturais, ocasionando a ressurgência da praga em níveis mais elevados. Uma possível solução para esse problema é o uso de inseticidas seletivos que preservem as populações de inimigos naturais (Pedigo, 1989).

As vespas Polybia fastidiosuscula Saussure, Polybia scutellaris (White) e Protonectarina sylveirae (Saussure) (Hymenoptera: Vespidae) constituem eficientes agentes do controle biológico natural de $D$. juno juno, predando grande quantidade de lagartas (Ruggiero, 1980). Apesar da importância dos Hymenoptera: Vespidae para o manejo integrado de pragas, são escassos os trabalhos nas áreas de ecologia (Rodrigues \& Morães, 1981), comportamento (Malaspina et al., 1990, 1992) e seletividade de inseticidas (Hebling-Beraldo et al., 1981; Picanço et al., 1998) com este grupo de predadores.

Este trabalho teve como objetivo o estudo da seletividade dos inseticidas cartape, deltametrina, fentiom e malatiom aos Hymenoptera: Vespidae P. fastidiosuscula, P. scutellaris e P. sylveirae, predadores de D. juno juno.

\section{MATERIAL E MÉTODOS}

Este trabalho foi conduzido no laboratório de manejo integrado de pragas da Universidade Federal de Viçosa (UFV), em Viçosa, MG, durante os anos, 1996/97. Os inseticidas utilizados foram cartape $500 \mathrm{PM}$, deltametrina $25 \mathrm{CE}$, fentiom $500 \mathrm{CE}$, e malatiom $500 \mathrm{CE}$. Utilizaram-se lagartas de quinto ínstar de Dione juno juno e adultos dos predadores Polybia fastidiosuscula, Polybia scutellaris e Protonectarina sylveirae (Hymenoptera: Vespidae) coletados no campus da UFV.

O delineamento experimental foi inteiramente casualizado, com quatro repetições. Para instalação do experimento, folhas de maracujá foram imersas, por cinco segundos, em soluções com seis concentrações de cada inseticida e em água (testemunha). Em todos os tratamentos empregou-se espalhante adesivo na dosagem de $30 \mathrm{~mL} / 100 \mathrm{~L}$ de calda (Andrei, 1996). As concentrações mínimas e máximas utilizadas foram: cartape 500 PM 0,1 a $0,7 \mathrm{mg}$ de ingrediente ativo (i.a.) $/ \mathrm{mL}$, deltametrina $25 \mathrm{CE} 0,01$ a $0,1 \mathrm{mg}$ de i.a. $/ \mathrm{mL}$, fentiom $500 \mathrm{CE} 0,005$ a $0,04 \mathrm{mg}$ de i.a. $/ \mathrm{mL}$ e malatiom $500 \mathrm{CE} 0,023$ a 1,3 mg de
i.a./mL. As folhas foram colocadas para secar por duas horas, e após a secagem foram acondicionadas em placas de Petri $(9 \mathrm{~cm}$ de diâmetro por $2 \mathrm{~cm}$ de altura). Em cada placa foram liberados 10 insetos. Assim, as vespas foram expostas aos inseticidas por contato. Já as lagartas de D. juno juno, além do contato, foram expostas também por ingestão, pois estas foram alimentadas com folhas de maracujá contendo inseticida. As placas de Petri foram levadas para estufa incubadora à temperatura de $25 \pm 0,5^{\circ} \mathrm{C}$ e umidade relativa de $75 \pm 5 \%$. Vinte e quatro horas depois, realizaram-se as avaliações da mortalidade dos insetos, e os resultados foram corrigidos em relação à mortalidade ocorrida na testemunha (Abbott, 1925). Determinaram-se, por análise de próbite, curvas concentração-mortalidade dos inseticidas em relação a $D$. juno juno e em relação às três espécies de vespas predadoras, e, por meio destas, foram estimadas as $\mathrm{CL}_{90}$ (concentração letal do inseticida em $90 \%$ dos indivíduos), sendo que foram aceitas curvas cuja probabilidade de aceitação do $\chi^{2}$ fosse maior que 0,05 (Finney, 1972).

Foram calculados os índices de seletividade diferencial $\left(\mathrm{ISD}_{90}=\mathrm{CL}_{90}\right.$ do inseticida em relação ao predador $/ \mathrm{CL}_{90}$ do inseticida em relação à praga), de toxicidade relativa $\left(\mathrm{ITR}_{90}=\right.$ maior $\mathrm{CL}_{90}$ em relação à espécie $/ \mathrm{CL}_{90}$ do inseticida em relação à espécie) e de tolerância relativa de P. fastidiosuscula e $P$. scutellaris em relação a $P$. sylveirae $\left(\right.$ ITRe $_{90}=\mathrm{CL}_{90}$ do inseticida em relação a uma das duas primeiras espécies/pela $\mathrm{CL}_{90}$ do inseticida em relação à P. sylveirae) (Alves et al., 1992; Batalha et al., 1995).

\section{RESULTADOS E DISCUSSÃO}

A deltametrina foi altamente seletiva aos predadores Polybia scutellaris $\left(\right.$ ISD $\left._{90}=104,94\right) \mathrm{e}$ Polybia fastidiosuscula $\left(\mathrm{ISD}_{90}=32,50\right)$, e medianamente seletiva à Protonectarina sylveirae $\left(\mathrm{ISD}_{90}=3,38\right)$ (Tabela 1). Guedes et al. (1992), Faleiro et al. (1995) e Picanço et al. (1997) verificaram que a deltametrina foi seletiva, respectivamente aos predadores Podisus nigrispinus (= connexivus) (Dallas) (Heteroptera: Pentatomidae), Doru luteipes (Scudder) (Dermaptera: Forficulidae) e Polybia ignobilis (Haliday) (Hymenoptera: Vespidae).

A concentração do cartape que ocasionou $90 \%$ de mortalidade a lagartas de Dione juno juno foi $2,29,5,29$ e 5,36 vezes menor do que a concentração que ocasionou a mesma mortalidade aos predadores P. scutellaris, P. sylveirae e 
P. fastidiosuscula respectivamente (Tabelas 1, 2 e 3). Assim, este inseticida pode ser considerado como mediamente seletivo aos três predadores. Leite et al. (1998) verificaram que o cartape foi seletivo a Brachygastra lecheguana (Latreille) (Hymenoptera: Vespidae).

O malatiom não apresentou seletividade a P. scutellaris $\left(\operatorname{ISD}_{90}=0,13\right)$, foi mediamente seletivo a $P$. fastidiosuscula ( ISD $\left._{90}=4,50\right) \mathrm{e}$ altamente seletivo a $P$. sylveirae $\left(\mathrm{ISD}_{90}=314,60\right)$ (Tabela 1). Suinaga et al. (1996) verificaram que o malatiom foi pouco seletivo ao predador P. nigrispinus.

O fentiom não apresentou seletividade a nenhum dos predadores, sendo sua $\mathrm{CL}_{90}$ 25, 100 e 100 vezes maior para $P$. scutellaris, $P$. sylveirae e

TABELA 1. Índice de seletividade diferencial $\left(\mathrm{ISD}_{90}\right)$ de quatro inseticidas utilizados no controle de Dione juno juno em relação aos predadores Polybia scutellaris, Protonectarina sylveirae e Polybia fastidiosuscula. Viçosa, MG, 1996/7.

\begin{tabular}{lccc}
\hline Inseticida & \multicolumn{3}{c}{ ISD $_{90}{ }^{1}$} \\
\cline { 2 - 4 } & P. scutellaris & P. sylveirae & P. fastidiosuscula \\
\hline Deltametrina & 104,94 & 3,38 & 32,50 \\
Cartape & 2,29 & 5,29 & 5,36 \\
Fentiom & 0,04 & 0,01 & 0,01 \\
Malatiom & 0,13 & 314,60 & 4,50 \\
\hline
\end{tabular}

${ }^{1} \mathrm{ISD}_{90}=\mathrm{CL}_{90}$ do inseticida em relação ao inimigo natural $/ \mathrm{CL}_{90}$ do inseticida em relação a lagartas de $D$. juno juno.

TABELA 2. Equação da curva concentração-mortalidade e concentração letal $\left(\mathbf{C L}_{90}\right)$ de quatro inseticidas em $\mathbf{9 0 \%}$ de larvas de último ínstar Dione juno juno e de adultos dos predadores Polybia fastidiosuscula, Polybia scutellaris e Protonectarina sylveirae. Viçosa, MG, 1996/7.

\begin{tabular}{|c|c|c|c|c|}
\hline Inseticida & Equação $^{1}$ & $\mathrm{CL}_{90}$ & $\chi^{2}$ & Probabilidade \\
\hline \multicolumn{5}{|c|}{ D. juno juno } \\
\hline Deltametrina & $Y^{\prime}=14,50+2,93 X$ & 0,0016 & 0,693 & 0,7124 \\
\hline Cartape & $Y^{\prime}=8,40+2,63 \mathrm{X}$ & 0,1563 & 0,457 & 0,9275 \\
\hline Fentiom & $Y^{\prime}=6,98+1,98 X$ & 0,4418 & 0,543 & 0,7662 \\
\hline Malatiom & $Y^{\prime}=7,17+2,23 X$ & 0,3994 & 0,351 & 0,8406 \\
\hline \multicolumn{5}{|c|}{ P. fastidiosuscula } \\
\hline Deltametrina & $Y^{\prime}=9,53+2,53 X$ & 0,0520 & 1,865 & 0,6014 \\
\hline Cartape & $\mathrm{Y}^{\prime}=6,58+3,90 \mathrm{X}$ & 0,8377 & 8,519 & 0,1286 \\
\hline Fentiom & $\mathrm{Y}^{\prime}=11,30+2,30 \mathrm{X}$ & 0,0066 & 4,581 & 0,0989 \\
\hline Malatiom & $Y^{\prime}=5,91+1,44 X$ & 1,7976 & 0,110 & 0,9459 \\
\hline \multicolumn{5}{|c|}{ P. scutellaris } \\
\hline Deltametrina & $Y^{\prime}=8,26+2,55 X$ & 0,1679 & 7,095 & 0,0677 \\
\hline Cartape & $Y^{\prime}=8,29+4,51 \mathrm{X}$ & 0,3580 & 7,524 & 0,1093 \\
\hline Fentiom & $Y^{\prime}=13,76+4,13 X$ & 0,0155 & 1,145 & 0,5697 \\
\hline Malatiom & $Y^{\prime}=9,63+2,62 X$ & 0,0528 & 2,724 & 0,2550 \\
\hline \multicolumn{5}{|c|}{ P. sylveirae } \\
\hline Deltametrina & $Y^{\prime}=14,14+3,47 X$ & 0,0054 & 6,325 & 0,1747 \\
\hline Cartape & $Y^{\prime}=6,67+4,70 X$ & 0,8265 & 5,276 & 0,3834 \\
\hline Fentiom & $Y^{\prime}=12,43+2,55 X$ & 0,0039 & 0,461 & 0,7968 \\
\hline Malatiom & $Y^{\prime}=0,48+2,76 X$ & 125,6493 & 0,635 & 0,7327 \\
\hline
\end{tabular}

${ }^{1} \mathrm{Y}^{\prime}$ : mortalidade em próbite; $\mathrm{X}$ : logaritmo da concentração $(\mathrm{mg} / \mathrm{mL})$. 
P. fastidiosuscula do que para as lagartas de Dione juno juno (Tabelas 1, 2 e 3). Picanço et al. (1996) verificaram que o fentiom também não apresentou seletividade aos predadores $P$. nigrispinus e Supputius cincticeps Stal (Heteroptera: Pentatomidae).

A seletividade da deltametrina e do cartape às três espécies de Vespidae e do malatiom a $P$. fastidiosuscula e $P$. sylveirae talvez se deva à menor sensibilidade do sítio de ação destes inseticidas nos predadores do que em sua presa, ou a menor taxa de penetração desses na cutícula dos Vespidae do que nas lagartas de D. juno juno (Suinaga et al., 1996). A seletividade da deltametrina pode também estar relacionada a maior taxa de metabolização deste piretróide por oxidases microssomais e esterases nos predadores do que nas lagartas de D. juno juno, acarretando maior desintoxicação nas vespas do que em sua presa (Yu, 1987, 1988).
P. fastidiosuscula e $P$. scutellaris foram cerca de 10 e 31 vezes mais tolerantes à deltametrina, e duas e quatro vezes mais tolerantes ao fentiom do que $P$. sylveirae, respectivamente. $P$. fastidiosuscula e $P$. sylveirae foram cerca de duas vezes mais tolerantes ao cartape do que $P$. scutellaris (Tabela 4). Suinaga et al. (1996) observaram que ninfas de terceiro ínstar do predador $P$. nigrispinus foram menos tolerantes à deltametrina do que ninfas de quinto ínstar e adultos.

$P$. sylveirae foi cerca de 70 e 2.500 vezes mais tolerante ao malatiom do que $P$. fastidiosuscula e P. scutellaris, respectivamente (Tabela 4). Picanço et al. (1996) observaram que adultos do predador P. nigrispinus foram mais tolerantes ao malatiom que $S$. cincticeps. Suinaga et al. (1996) verificaram que ninfas de quinto ínstar do predador P. nigrispinus foram mais tolerantes ao malatiom que ninfas de terceiro ínstar e adultos.

TABELA 3. Índice de toxicidade relativa (ITR) de quatro inseticidas a lagartas de Dione juno juno e aos seus predadores Polybia fastidiosuscula, Polybia scutellaris e Protonectarina sylveirae. Viçosa, MG, 1996/7.

\begin{tabular}{lcccc}
\hline Inseticida & \multicolumn{4}{c}{ ITR $_{90}{ }^{1}$} \\
\cline { 2 - 5 } & D. juno juno & P. fastidiosuscula & P. scutellaris & P. sylveirae \\
\hline Deltametrina & 276,12 & 34,57 & 2,13 & 23268,39 \\
Cartape & 2,83 & 2,15 & 1,00 & 152,03 \\
Fentiom & 1,00 & 272,36 & 23,10 & 32217,77 \\
Malatiom & 1,11 & 1,00 & 6,78 & 1,00 \\
\hline${ }^{1} \mathrm{ITR}_{90}=$ razão da maior $\mathrm{CL}_{90}$ em relação à espécie/ $\mathrm{CL}_{90}$ do inseticida em relação à espécie.
\end{tabular}

TABELA 4. Índice de tolerância relativo (ITRe ${ }_{90}$ ) de quatro inseticidas para os predadores Polybia fastidiosuscula e Polybia scutellaris em relação à Protonectarina sylveirae. Viçosa, MG, 1996/7¹.

\begin{tabular}{lcc}
\hline Inseticida & P. fastidiosuscula/ P. sylveirae & P. scutellaris/ P. sylveirae \\
\hline Deltametrina & 9,6296 & 31,0926 \\
Cartape & 1,0136 & 0,4332 \\
Fentiom & 1,6923 & 3,9744 \\
Malatiom & 0,0143 & 0,0004 \\
\hline
\end{tabular}

${ }^{1} \mathrm{ITRe}_{90}=\mathrm{CL}_{90}$ do inseticida para P. fastidiosuscula ou P. scutellaris dividida pela $\mathrm{CL}_{90}$ do inseticida para P. sylveirae. 
Em função da suscetibilidade relativa das vespas aos inseticidas, em pomares de maracujá com intensa aplicação de inseticidas, deve-se conhecer com profundidade as espécies de vespas predadoras presentes. Em função dessa informação, deve-se recomendar os inseticidas mais seletivos a elas.

As curvas concentração-mortalidade do cartape em relação à $P$. scutellaris, $P$. fastidiosuscula e
P. sylveirae, da deltametrina em relação à $P$. sylveirae e do fentiom em relação às três espécies de vespas apresentaram maiores inclinações do que as curvas destes inseticidas em relação a lagartas de $D$. juno juno (Tabela 2 e Fig. 1). Esses dados indicam que pequenas variações nas dosagens destes inseticidas ocasionam maiores aumentos nas mortalidades
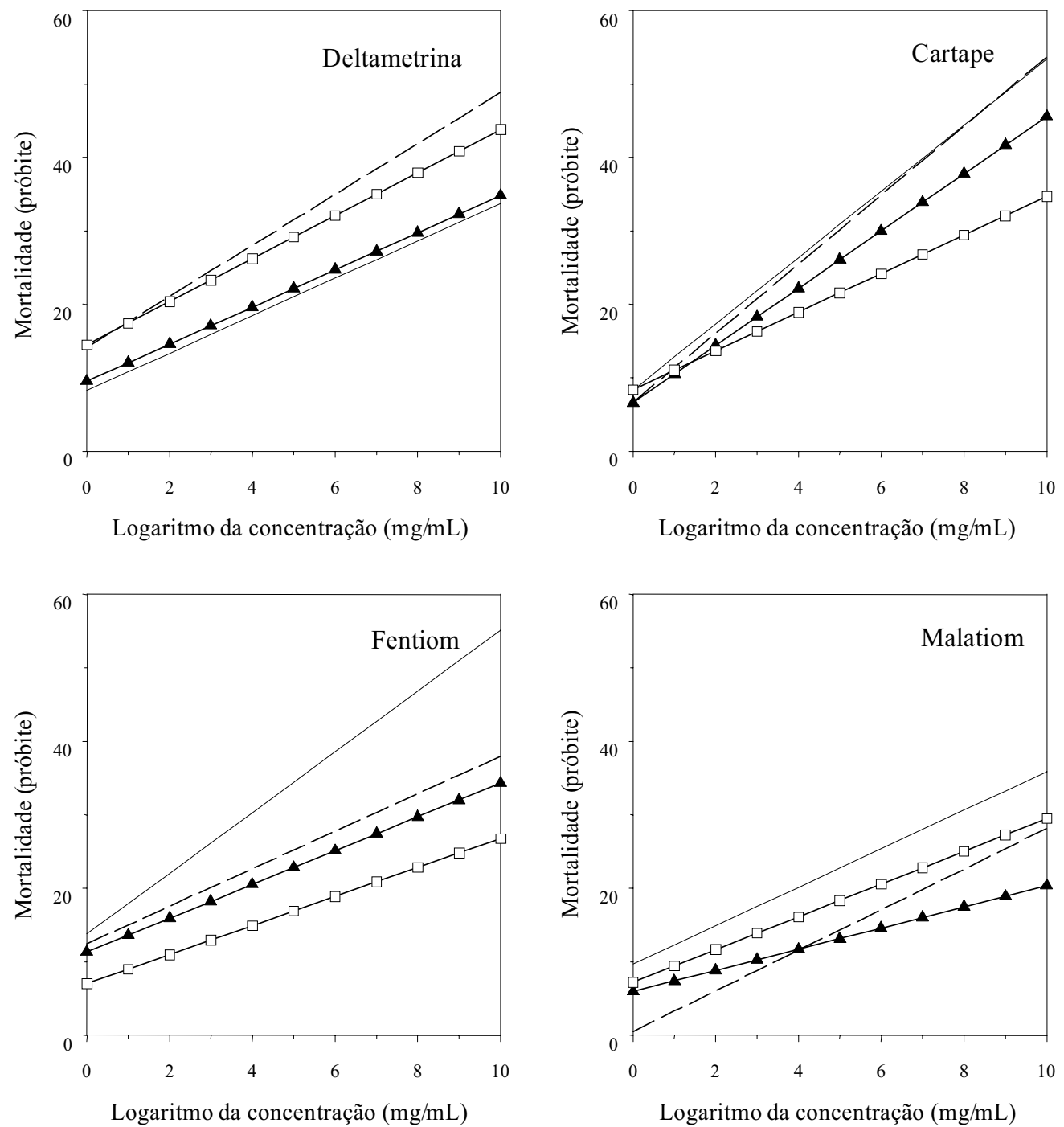

FIG. 1. Curvas concentração-mortalidade de quatro inseticidas em relação a adultos de Polybia

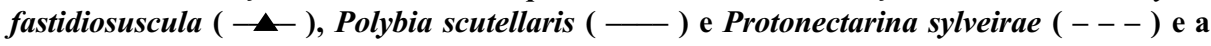
larvas de último ínstar Dione juno juno ( $\square-$ ). Viçosa, MG, 1996/7. 
destes predadores do que nas das lagartas de D. juno juno. Assim, erros na calibração de dosagens destes inseticidas poderão ocasionar maiores problemas a estes predadores do que a lagartas de D. juno juno (Atkins et al., 1973).

\section{CONCLUSÕES}

1. A deltametrina é seletiva às vespas predadoras Polybia scutellaris e Polybia fastidiosuscula e medianamente seletiva à Protonectarina sylveirae.

2. O cartape é medianamente seletivo às três espécies de vespas predadoras.

3. O malatiom é seletivo às vespas predadoras $P$. sylveirae e medianamente seletivo a P. fastidiosuscula.

4. As vespas $P$. fastidiosuscula e $P$. scutellaris são mais tolerantes à deltametrina e ao fentiom do que $P$. sylveirae; $P$. fastidiosuscula e $P$. sylveirae são mais tolerantes ao cartape do que $P$. scutellaris; $P$. sylveirae é mais tolerante ao malatiom do que $P$. fastidiosuscula e P. scutellaris.

\section{REFERÊNCIAS}

ABBOTT, W.S. A method of computing the effectiveness of insecticide. Journal of Economic Entomology, v.18, n.4, p.265-267, 1925.

ALVES, P.M.P.; LIMA, J.O.G.; OLIVEIRA, L.M Monitoramento da resistência do bicho mineiro-docafeeiro, Perileucoptera coffeella (Lepidoptera: Lyonetiidae) a inseticidas em Minas Gerais. Anais da Sociedade Entomológica do Brasil, v.21, n.2, p.77-91, 1992.

ANDREI, E. Compêndio de defensivos agrícolas. 5.ed. São Paulo : Andrei, 1996. 506p.

ATKINS, E.L.; GERYWOOD E.A.; MACDONALD, R.L. Toxicity of pesticides and other agricultural chemicals to honey bees. Laboratory studies. California : University of California, Agricultural Extension Service, 1973. 36p. (Technical bulletin, M-16).

BATALHA, V.C.; ZANUNCIO, J.C.; PICANÇO, M.C.; SEDIYAMA, C.S. Seletividade de inseticidas aos predadores Podisus nigrispinus (Dallas, 1851) e Supputius cincticeps (Stal, 1860) (Heteroptera:
Pentatomidae) e a sua presa Lepidoptera. Revista Árvore, v.19, n.3, p.382-395, 1995.

FALEIRO, F.G.; PICANÇO, M.C.; PAULA, S.V.; BATALHA, V.C. Seletividade de inseticidas a Spodoptera frugiperda (J.E. Smith) (Lepidoptera: Noctuidae) e ao predador Doru luteipes (Scudder) (Dermaptera: Forficulidae). Anais da Sociedade Entomológica do Brasil, v.24, n.2, p.247-252, 1995.

FINNEY, D.J. Probit analysis. 3.ed. London : Cambridge University, 1971. 333p.

GUEDES, R.N.C.; LIMA, J.O.G.; ZANUNCIO, J.C. Seletividade dos inseticidas deltametrina, fenvalerato e fenitrotiom para Podisus connexivus (Bergroth, 1891) (Heteroptera: Pentatomidae). Anais da Sociedade Entomológica do Brasil, v.21, n.3, p.339-346, 1992.

HEBLING-BERALDO, M.J.A.; ROCHA, E.A.; MACHADO, V.L.L. Toxicidade de inseticidas (em laboratório) para Polybia (Mirapetra) paulista (Ihering, 1896) (Hymenoptera - Vespidae). Anais da Sociedade Entomológica do Brasil, v.10, n.2, p.261-267, 1981.

LEITE, G.L.D.; PICANÇO, M.; GUEDES, R.N.C; GUSMÃO, M.R. Selectivity of insecticides with and without mineral oil to Brachygastra lecheguana a predator of Tuta absoluta. Ceiba, v.39, n.1, p.3-6, 1998.

LIMA, A.A.; BORGES A.L.; SANTOS FILHO, H.P.; SANTOS, L.B.; FANCELLI, M.; SANCHES. N.F. Instruções práticas para o cultivo do maracujazeiro. Cruz das Almas : Embrapa-CNPMF, 1994. p.26-33. (Embrapa-CNPMF. Circular técnica, 20).

MALASPINA, O.; GOBBI, N.; MACHADO, V.L.L. Capacidade de transporte de alimento de Polybia (Trichothorax) ignobilis (Haliday, 1936) (Hymenoptera: Vespidae). Anais da Sociedade Entomológica do Brasil, v.21, n.1, p.169-173, 1992.

MALASPINA, O.; GOBBI, N.; MACHADO, V.L.L. Capacidade de transporte de alimento em operárias de Polybia (Myrapetra) paulista Ihering, 1896 (Hymenoptera: Vespidae). Anais da Sociedade Entomológica do Brasil, v.19, n.2, p.457-463, 1990.

PEDIGO, L.P. Entomology and pest management. New York : MacMillan, 1989. 646p.

PICANÇO, M.C.; GUEDES, R.N.C.; BATALHA, V.C.; CAMPOS, R.P. Toxicity of insecticides to Dione 
juno juno (Lepidoptera: Heliconidae) and selectivity to two of its predaceous bugs. Tropical Science, v.36, n.1, p.51-53, 1996.

PICANÇO, M.; RIBEIRO, L.J.; LEITE, G.L.D.; GUSMÃO, M.R. Seletividade de inseticidas a Polybia ignobilis (Haliday) (Hymenoptera: Vespidae) predador de Ascia monuste orseis (Godart) (Lepidoptera: Pieridae). Anais da Sociedade Entomológica do Brasil, v.27, n.1, p.73-78, 1998.

PICANÇO, M.; RIBEIRO, L.J.; LEITE, G.L.D.; ZANUNCIO, J.C. Seletividade de inseticidas a Podisus nigrispinus predador de Ascia monuste orseis. Pesquisa Agropecuária Brasileira, Brasília, v.32, n.4, p.369-372, 1997.

RODRIGUES, V.M.; MORÃES, R.A.O. Vespídeos sociais - Estudo de Polybia (Apopolybia) jurinei De Saussure, 1854 (Polistinae: Polybiini). Anais da Sociedade Entomológica do Brasil, v.10, n.1, p.3-7, 1981.

RUGGIERO, C. Cultura do maracujazeiro. Jaboticabal : FCAV-UNESP, 1980. 147p.
SUINAGA, F.A.; PICANÇO, M.; ZANUNCIO, J.C.; BASTOS, C.S. Seletividade fisiológica de inseticidas a Podisus nigrispinus (Dallas, 1851) (Heteroptera: Pentatomidae) predador de lagartas desfolhadoras de eucalipto. Revista Árvore, v.20, n.3, p.407-414, 1996.

TEIXEIRA, C.G.; CASTRO, J.V.; TOCCHINI, R.P.; NISIDA, A.L.A.C.; HASHIZUME, T.; MEDINA, J.C.; TURATTI, J.M.; LEITE, R.S.S.F.; BLISKA, F.M.M.; GARCIA, A.E.B. Maracujá: cultura, matéria-prima, processamento e aspectos econômicos. 2.ed. Campinas : ITAL, 1994. 267p.

YU, S.J. Biochemical defense capacity in the spined soldader bug (Podisus maculiventris) and its lepidopterus prey. Pesticide Biochemistry and Physiology, v.28, n.1, p.216-223, 1987.

YU, S.J. Selectivity of insecticides to the spined soldader bug (Heteroptera: Pentatomidae) and its lepidopterus prey. Journal of Economic Entomology, v.81, n.1, p.119-222, 1988. 